\title{
Terahertz Radiation from a Plasma Cylinder with External Radial Electric and Axial Magnetic Fields
}

\author{
L. H. Cao $\mathbb{D}^{1,2}$ Wei Yu, ${ }^{3,4}$ M. Y. Yu, ${ }^{4,5,6}$ and C. Y. Yu ${ }^{7}$ \\ ${ }^{1}$ Institute of Applied Physics and Computational Mathematics, Beijing 100088, China \\ ${ }^{2}$ HEDPS, Center for Applied Physics and Technology, Peking University, Beijing 100871, China \\ ${ }^{3}$ Shanghai Institute of Optics and Fine Mechanics, Shanghai 201800, China \\ ${ }^{4}$ College of Engineering Physics, Shenzhen Technology University, Shenzhen 518118, China \\ ${ }^{5}$ Institute for Fusion Theory and Simulation, Zhejiang University, Hangzhou 310027, China \\ ${ }^{6}$ Institute for Theoretical Physics I, Ruhr University, D-44780 Bochum, Germany \\ ${ }^{7}$ State Key Laboratory of Drug Research, Shanghai Institute of Materials Media, Chinese Academy of Sciences, \\ Shanghai 201203, China \\ Correspondence should be addressed to L. H. Cao; cao_lihua@iapcm.ac.cn
}

Received 13 October 2020; Revised 4 December 2020; Accepted 14 December 2020; Published 30 January 2021

Academic Editor: Dieter H.H. Hoffmann

Copyright (C) $2021 \mathrm{~L}$. H. Cao et al. This is an open access article distributed under the Creative Commons Attribution License, which permits unrestricted use, distribution, and reproduction in any medium, provided the original work is properly cited.

\begin{abstract}
Terahertz $(\mathrm{THz})$ radiation from a plasma cylinder with embedded radial electric and axial magnetic fields is investigated. The plasma density and the electric and magnetic fields are such that the electron plasma frequency is near the electron cyclotron frequency and in the THz regime. Two-dimensional particle-in-cell simulations show that the plasma electrons oscillate not only in the azimuthal direction but also in the radial direction. Spectral analysis shows that the resulting oscillating current pattern has a clearly defined characteristic frequency near the electron cyclotron frequency, suggesting resonance between the cyclotron and plasma oscillations. The resulting far-field $\mathrm{THz}$ radiation in the axial direction is also discussed.
\end{abstract}

\section{Introduction}

The frequency regime of Terahertz $(\mathrm{THz})$ radiation lies between that of microwaves and optical waves. Because of shortage of effective sources, the $\mathrm{THz}$ regime was often considered as a gap in the usable electromagnetic-wave spectrum. Generation and manipulation of $\mathrm{THz}$ radiation (or T-rays) have been extensively investigated because of its relevance in many areas of basic science and application. In particular, it has potential use in medical and security-detection imaging and communication, as well as in investigating molecular dynamics in matter in the condensed phase.

Many approaches have been proposed for generating T-rays, including synchrotron radiation [1], optical transition radiation $[2,3]$, and nonlinear frequency upconversion [4]. Optical transition radiation is produced by relativistic electrons as they cross the interface of different dielectric media. Frequency up- or downconversion of electromagnetic radiation can be realized through nonlinear wavematter and wave-wave interaction. $\mathrm{THz}$ radiation can also be produced in intense laser interaction with matter [3, 5, 6-14]. T-rays can also be produced by the oscillating electrons in laser-induced wakefields [15-17], Smith-Purcell effect of the electron beams in laser-matter interactions [18], two-color laser gas-plasma interaction [19, 20], transitionCherenkov effect from laser filaments [21], laser-driven wire-guided helical undulators [22], and linear and nonlinear mode conversion [23], as well as laser interaction with strongly magnetized plasmas [24-27]. However, most such sources of T-rays are rather complex and inconvenient to realize in practice.

We consider in this paper an unusual but simple source of T-rays, namely, a plasma cylinder with embedded axial magnetic electric and radial fields. Two-dimensional (2D) particle-in-cell (PIC) simulations show that electrons in the 
plasma cylinder oscillate in the azimuthal as well as radial directions. The evolution of the oscillating current distribution is investigated. It is found that resonance-like behavior between the electron oscillations near the plasma and cyclotron frequencies can take place, and the resulting current pattern can produce T-rays in the axial direction.

This paper is organized as follows. In Section 2, the proposed scheme and simulation parameters are described. Section 3 presents the simulation results at the initial stage, and Section 4 considers the long-time behavior. The far-field power of the T-ray emitted from the plasma cylinder is given in Section 5. Section 6 gives the summary.

\section{Proposed Scheme and Simulation Parameters}

We consider here an unusual source of $\mathrm{THz}$ radiation, namely, from a plasma cylinder with external radial electric field and axial magnetic field, as shown in Figure 1. The electric field producing the plasma could be from a radial capacitor discharge and the magnetic field from a solenoid coil outside the cylinder. The electron motion is governed by

$$
\begin{aligned}
d_{t}(\gamma u) & =-e\left(E+\frac{1}{c} u \times B\right), \\
d_{t} \gamma & =\frac{e}{m c^{2}} u \times E,
\end{aligned}
$$

where $u$ is the electron velocity, $\gamma$ is the relativistic factor, and $e, m$, and $c$ are the electron charge and mass, and the speed of light, respectively. The dynamics of both electrons and (hydrogen) ions are fully included in the PIC simulations [28]. However, as expected, within the picosecond time of interest here, the electron motion is dominant. The constant embedded electric and magnetic fields are $E_{0}=E_{0} \widehat{r}$ and $B_{0}=B_{0} \widehat{z}$, where $E_{0}=3.4 \times 10^{10} \mathrm{~V} / \mathrm{m}$ and $B_{0}=11.4 \mathrm{~T}$, which we note are readily realizable. The cylindrical plasma cylinder is of radius $R=3286 \mu \mathrm{m}$ and initially of uniform density $n_{0}=1.26 \times 10^{20} \mathrm{~m}^{-3}$. The temperature of the electrons and ions is $300 \mathrm{eV}$. The parameters have been chosen such that the electron cyclotron frequency $\omega_{c}=e B_{0} / m c$ and plasma frequency $\omega_{p e}=\left(4 \pi e^{2} n_{0} / m\right)^{1 / 2}$ are both in the $\mathrm{THz}$ regime, so that resonance of the electron oscillations can be expected.

To investigate the evolution of such a plasma cylinder, we use the $2 \mathrm{D} 3 \mathrm{~V}$ (two-dimensional in space and threedimensional in velocity) PIC simulation code LAPINE [28]. The size of the simulation box is $9418 \mu \mathrm{m} \times 9418 \mu \mathrm{m}$. The spatial mesh has $1024 \times 1024$ cells, containing $4 \times 10^{7}$ each of electrons and ions, and the simulation time step is $13 \mathrm{fs}$. As mentioned, the ion dynamics is fully included, as they might play a role in the long-time behavior of the system.

\section{Initial Stage of the Evolution}

Figure 2 shows the self-consistently induced electric field $\Delta E_{r}$ at $t=13.18 \mathrm{ps}$ and $t=16.11 \mathrm{ps}$. In this beginning stage of the evolution, besides the cyclotron motion, the plasma electrons are driven radially by external electric field $E_{0}$ and

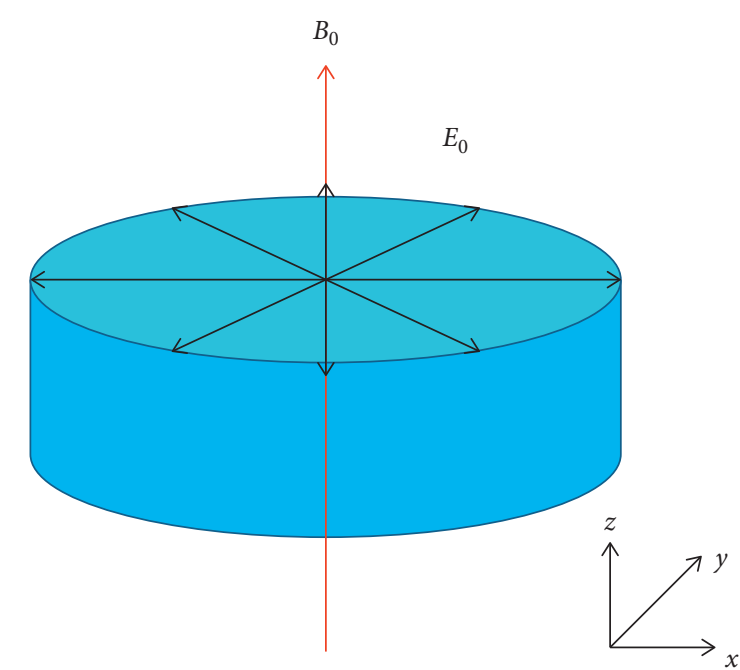

Figure 1: The plasma cylinder with external radial electric field $E_{0}$ and axial magnetic field $B_{0}$, where $E_{0}=3.4 \times 10^{10} \mathrm{~V} / \mathrm{m}$ and $B_{0}=11.4 \mathrm{~T}$. The (hydrogen) plasma density is $n_{0}=1.26 \times 10^{20} \mathrm{~m}^{-3}$.

acquire a radial velocity $v_{r}$. The induced electric field $\Delta E_{r}$, of order hundreds $\mathrm{V} / \mathrm{m}$, is much weaker than $E_{0}$. The Lorentz force from $B_{0}$ and $v_{r}$ also cause the electrons to move in the azimuthal direction, and the resulting azimuthal velocity in turn causes the electrons to move in the radial direction, leading to the oscillations. The much heavier ions have hardly moved (not shown).

The evolution of the current density distribution in the early stage, involving about two oscillation periods, is shown in Figure 3. Figure 3(a) shows that, at $t=11.72 \mathrm{ps}$, the current density near the cylinder center is larger than that near the boundary. Furthermore, the local current vectors (black arrows) indicate that the stronger current is in the anticlockwise direction (note that the electrons near the center rotate in the clockwise direction). For $t=13.18 \mathrm{ps}$, Figure 3(b) shows that the outward-moving electrons approach the boundary of the circular column. They are then reflected by the sheath there and move inwards toward the center, as can be seen in Figure 3(c). Roughly, Figures 3(a) and $3(\mathrm{c})$ correspond to near-minimum amplitudes and Figures 3(b) and 3(d) to near-maximum amplitudes, of the current oscillations. The evolution of the current density in Figure 3 shows that besides rotating in the anticlockwise direction, the electrons oscillate in a rather complicated manner.

\section{Long-Time Behavior}

Figure 3 shows the electron dynamics in the initial two oscillation cycles. To see the behavior at longer times, we now focus on a fixed location inside the plasma cylinder. Figures 4(a) and 4(c) show the evolution of the radial and azimuthal currents at $(x, y)=(7346.04 \mu \mathrm{m}, 7346.04 \mu \mathrm{m})$. One can see that, during the 80 ps long interval, the electron currents $J_{r}$ and $J_{\varphi}$ have undergone many oscillation cycles. Figures 4(b) and 4(d) show the 


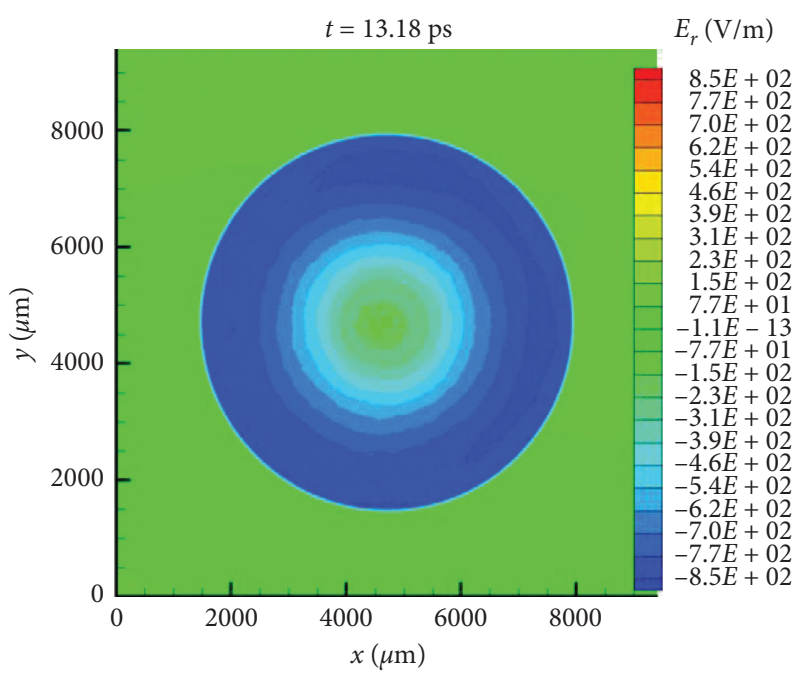

(a)

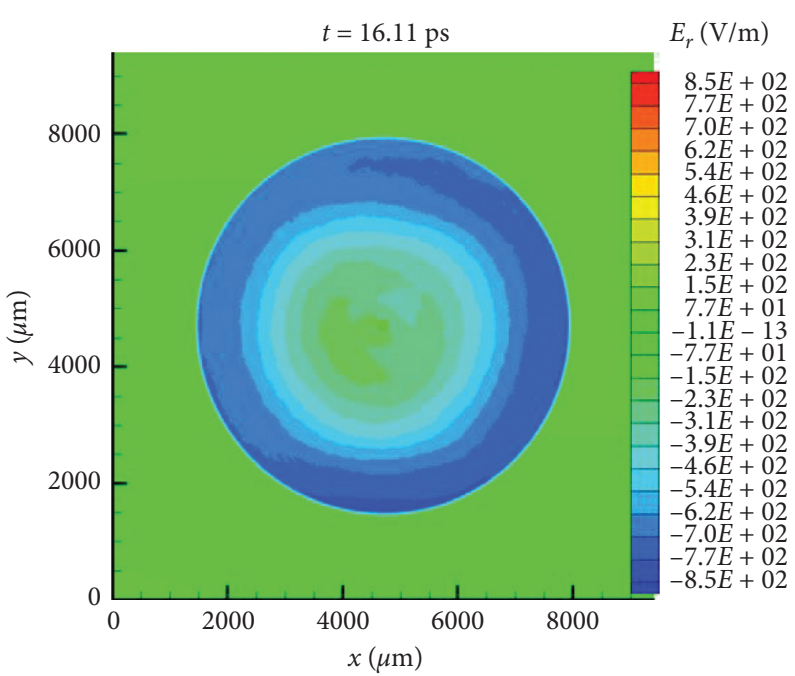

(b)

FIGURE 2: Snapshots of the induced electric field $\Delta E_{r}$ at (a) $t=13.18 \mathrm{ps}$ and (b) $t=16.11 \mathrm{ps}$. Note that the induced electric field is much weaker than the embedded electric field.

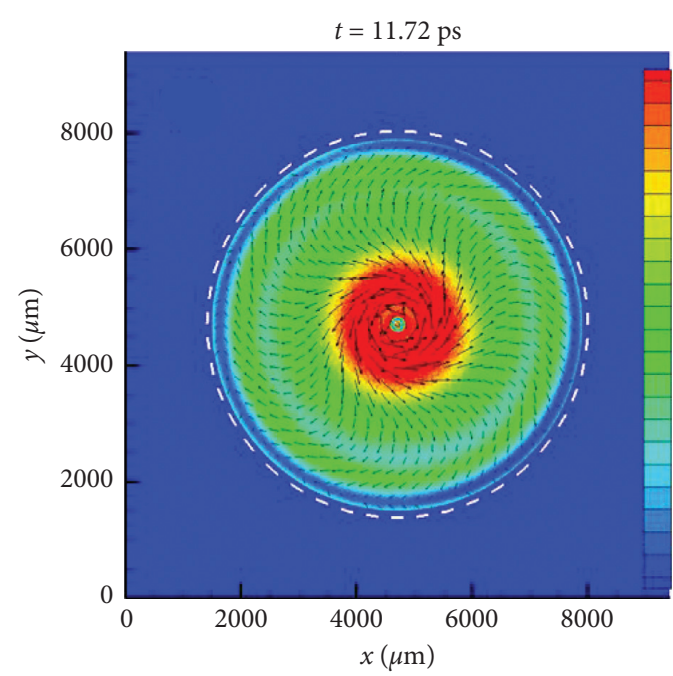

(a)

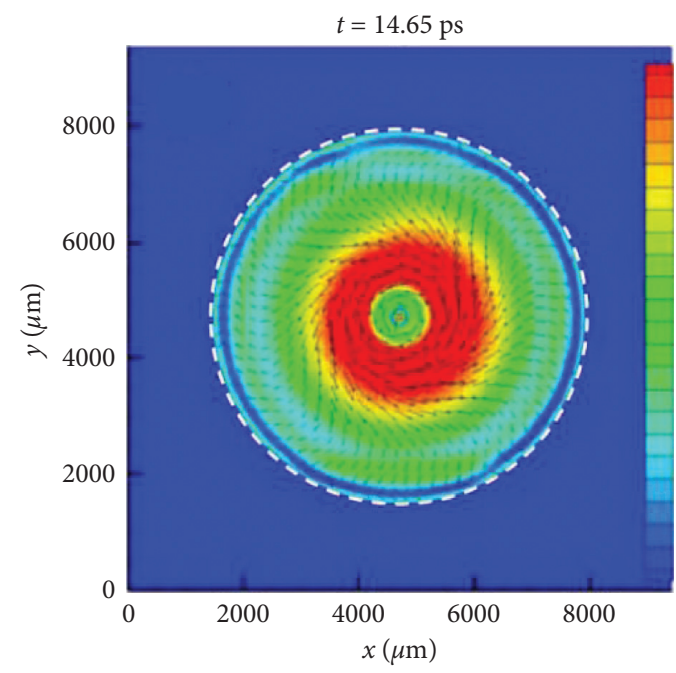

(c)
$J\left(\mathrm{~A} / \mathrm{m}^{2}\right)$

$4.0 \mathrm{E}-03$ $3.8 E-03$ $3.7 E-03$
$3.5 E-03$ $3.3 E-03$ $3.3 E-03$
$3.2 E-03$ $3.0 E-03$
$2.9 E-03$ $2.9 E-03$ $2.7 E-03$
$2.5 E-03$ $2.4 E-03$ $2.2 E-03$ $2.0 E-03$ $1.9 E-03$ $1.5 E-03$ $1.4 E-03$ $1.1 E-03$ $8.9 E-04$ $8.9 E-04$
$7.3 E-04$ $5.6 E-04$ $4.0 E-04$ $J\left(\mathrm{~A} / \mathrm{m}^{2}\right)$ $4.0 E-03$
$3.8 E-03$
$3.7 E-03$
$3.5 E-03$
$3.3 E-03$
$3.2 E-03$
$3.0 E-03$
$2.9 E-03$
$2.7 E-03$
$2.5 E-03$
$2.4 E-03$
$2.2 E-03$
$2.0 E-03$
$1.9 E-03$
$1.7 E-03$
$1.5 E-03$
$1.4 E-03$
$1.2 E-03$
$1.1 E-03$
$8.9 E-04$
$7.3 E-04$
$5.6 E-04$
$4.0 E-04$ (c)

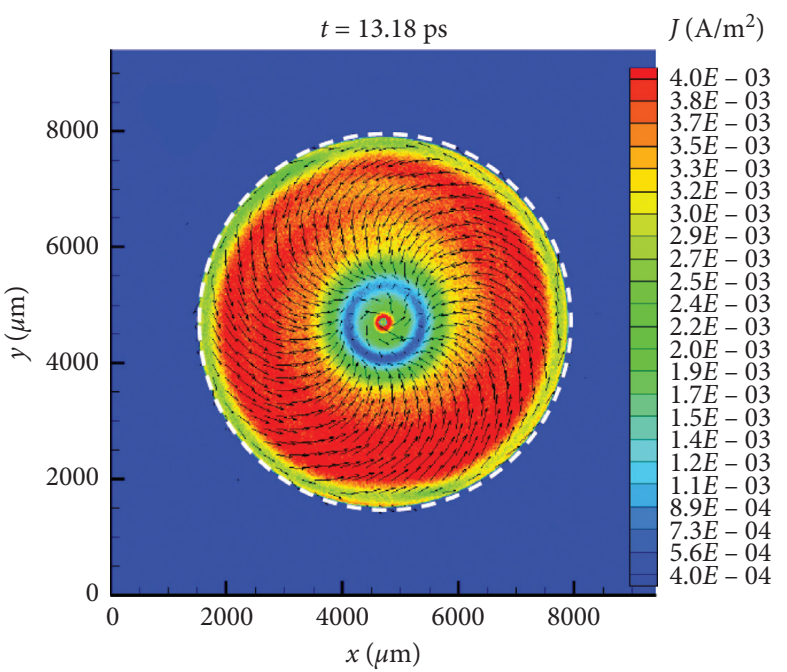

(b)

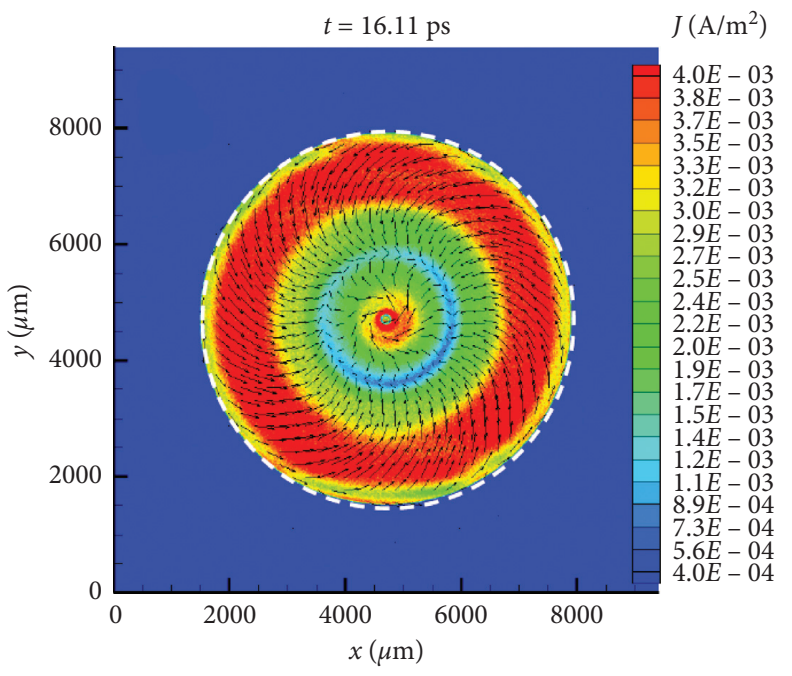

(d)

Figure 3: Snapshots of the current distribution at (a) $t=11.72$, (b) $t=13.18$, (c) $t=14.65$, and (d) $t=16.11$ ps. The black arrows represent local current vectors. Close inspection shows that the currents oscillate both radially and azimuthally. The white dashed circles mark the boundary of the initial plasma cylinder. 


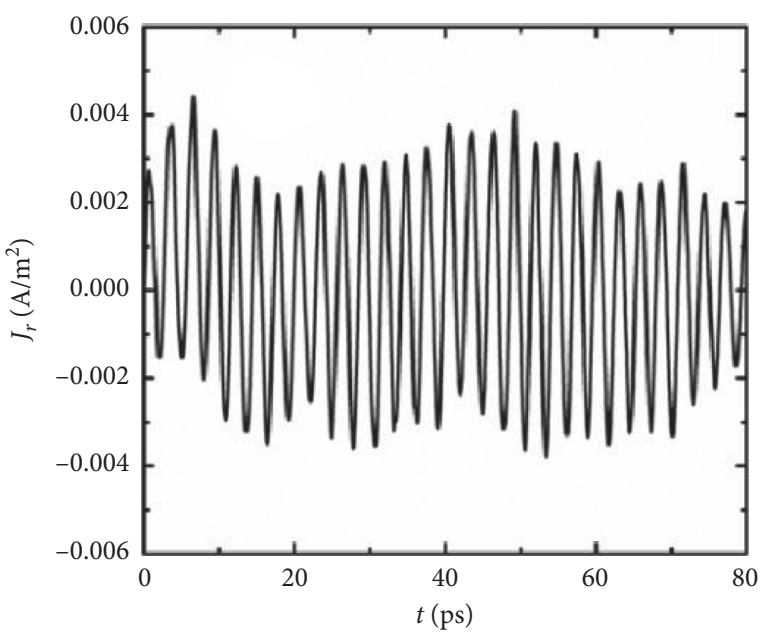

(a)

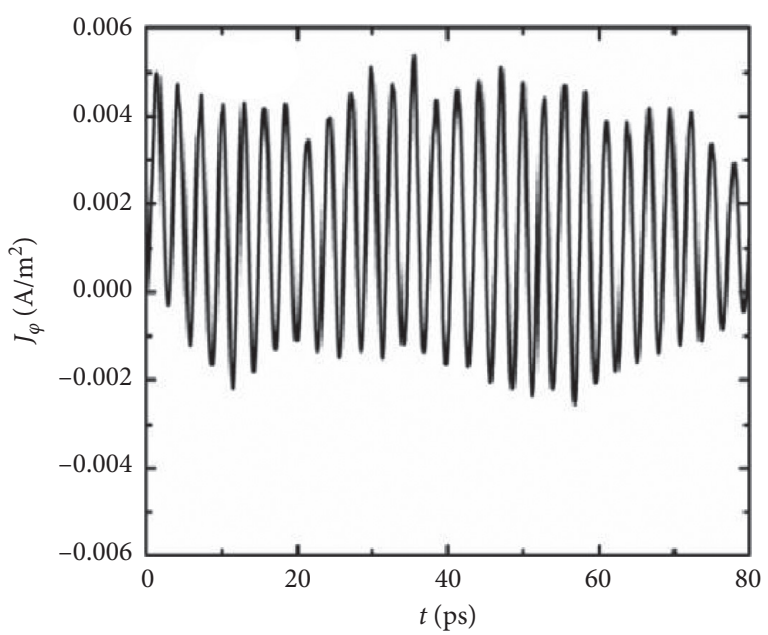

(c)

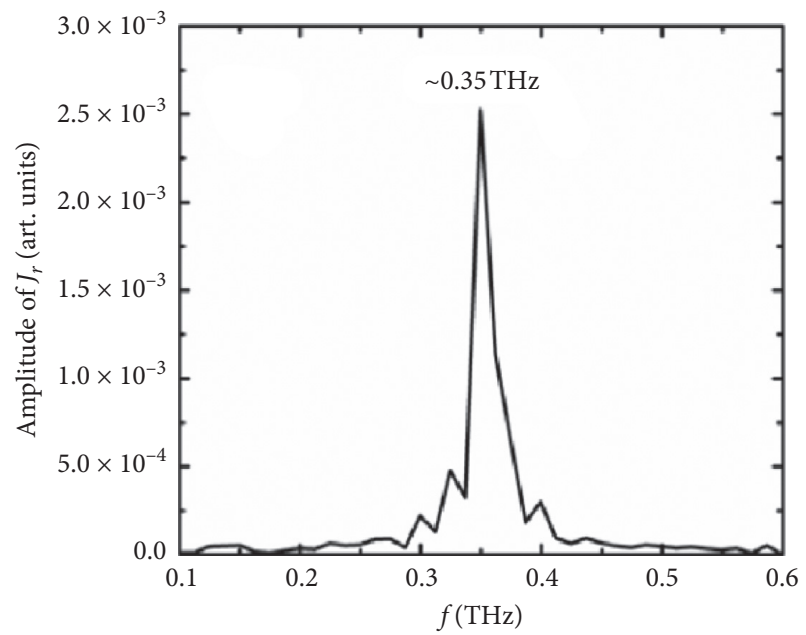

(b)

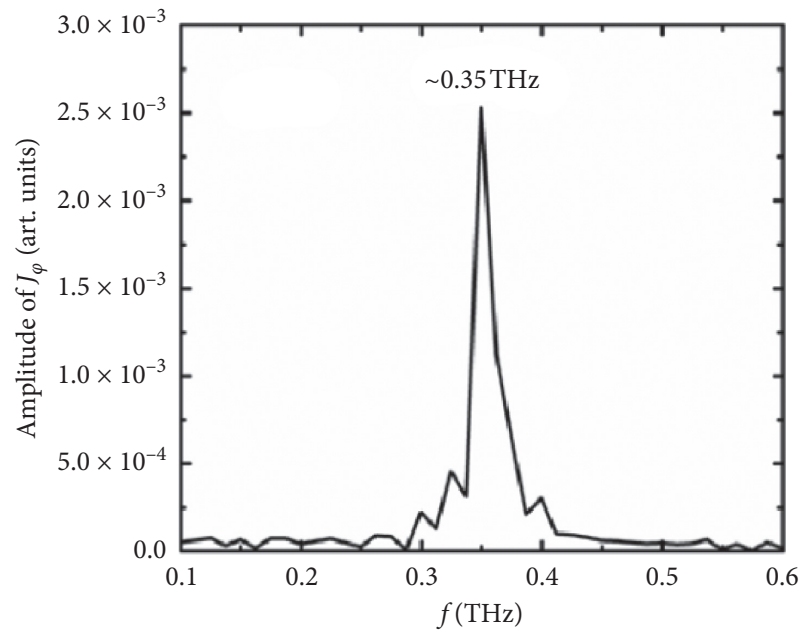

(d)

Figure 4: Evolution of the (a) radial current density $J_{r}$ and (c) azimuthal current density $J_{\varphi}$ at $(x, y)=(7346.04 \mu \mathrm{m}, 7346.04 \mu \mathrm{m})$. (b, d) The corresponding frequency spectra.

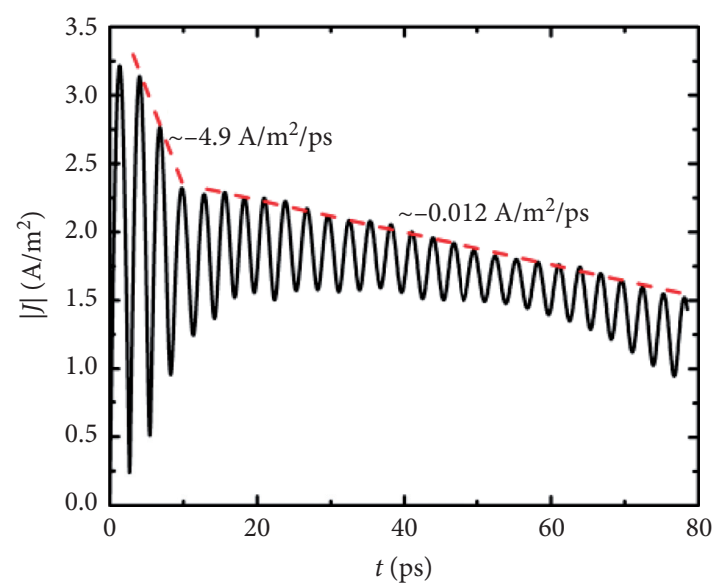

(a)

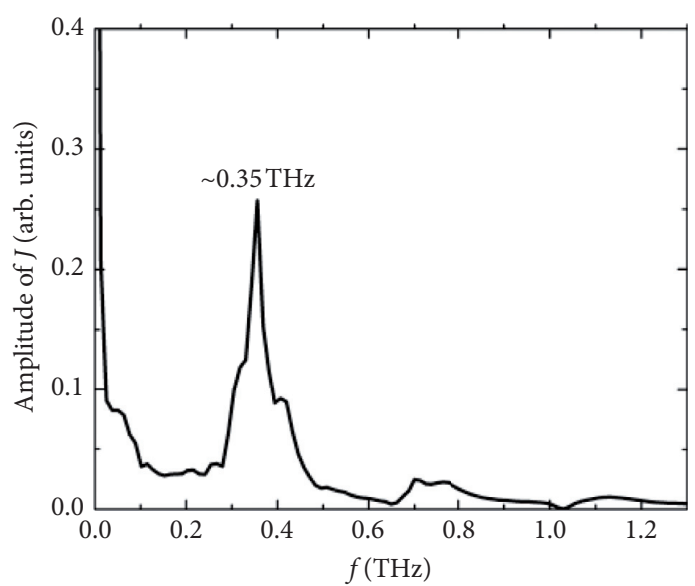

(b)

FIGURE 5: Evolution of the (a) total current density $\sum|J(x)|$ and (b) its frequency spectrum. 


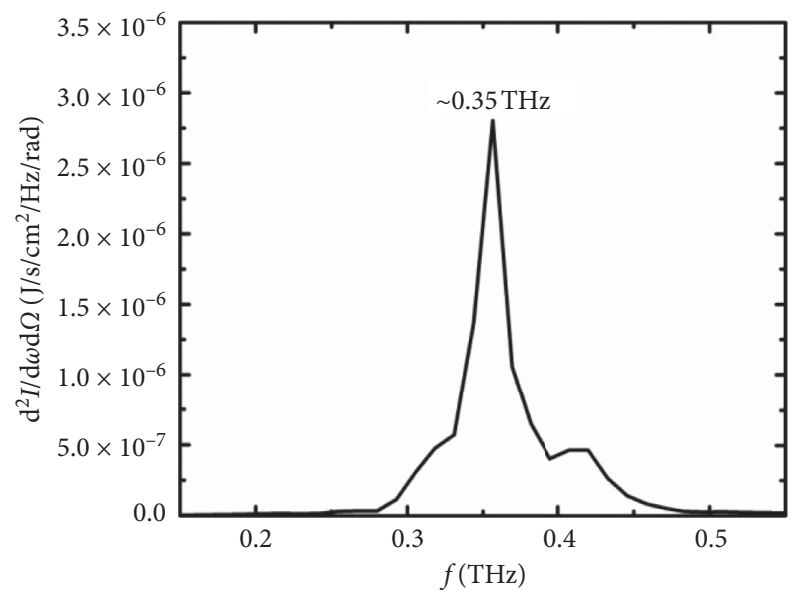

FIGURE 6: The frequency spectrum of the THz radiation. The peak is at $\sim 0.35 \mathrm{THz}$, and the bandwidth is $\sim 5.7 \%$.

corresponding frequency spectra. We can see in both the radial and azimuthal spectra a dominant peak at $f \sim 0.35 \mathrm{THz}$ (or $\omega \sim 2.2 \mathrm{rad} / \mathrm{sec}$ ). In fact, their overall spectral profiles are almost identical, suggesting that a $2 \mathrm{D}$ normal-mode-like structure has formed.

Figure 5 shows the evolution of the total current density $\sum|J(x)|$ in the simulation box. Figure 5(a) shows that the initial oscillations of the total current density are of large amplitude (to be expected since the initial plasma and fields do not form a self-consistent system), and the corresponding rate of decrease of the envelope is $-4.9 \mathrm{~A} / \mathrm{m}^{2} / \mathrm{ps}$. At later times, the rate of decrease becomes $-0.0012 \mathrm{~A} / \mathrm{m}^{2} / \mathrm{ps}$. Figure $5(\mathrm{~b})$ shows the corresponding frequency spectrum, showing that the characteristic frequency of the current oscillations at this location is also $\sim 0.35 \mathrm{THz}$.

\section{Terahertz Radiation}

Using the temporal and spatial current density from the simulation, one can obtain the far-field radiation. The total electromagnetic energy per unit solid angle $\Omega$ per unit frequency is given by the following equation $[15,29]$ :

$$
\frac{\mathrm{d}^{2} I}{\mathrm{~d} \omega \mathrm{d} \Omega} \approx \frac{\omega^{2}}{4 \pi^{2} c^{3}}\left|\int \mathrm{d} t \int d^{3} x \widehat{n} \times[\widehat{n} \times J(x, t)] e^{i \omega(t-\hat{n} \cdot x / c)}\right|^{2},
$$

where $\hat{n}$ is the direction of the observer with respect to the radiating body. The intensity distribution of the radiation in the $z$-direction is then [15]

$$
\frac{d^{2} I}{\mathrm{~d} \omega \mathrm{d} \Omega} \approx \frac{\omega^{2}}{4 \pi^{2} c^{3}}\left|\int \mathrm{d} t J(t) e^{i \omega t}\right|^{2},
$$

where $J(t)=\int d^{3} x J(x, t)$. The corresponding result is shown in Figure 6. We can see that the radiation spectrum has a broad peak centered at $f_{0} \sim 0.35 \mathrm{THz}$, as to be expected in view of the current density spectra discussed above. The full-width-at-half-maximum bandwidth $\Delta f / f_{0}$ is about 0.057 .

\section{Summary}

In this paper, we have shown that a bounded plasma cylinder with embedded radial electric and axial magnetic fields can emit T-rays if the parameters of the plasma and the electric and magnetic fields are such that the electron plasma and cyclotron frequencies nearly match and are in the $\mathrm{THz}$ regime. As a result, resonance-like behavior of the electrons can occur. The electrons not only rotate in the azimuthal direction but also oscillate in both the radial and azimuthal directions. The resulting currents can emit THz radiation. Finally, it may be of interest to note that, in a nonperturbative analysis of the cold fluid and Maxwell equations, where all the electric and magnetic fields are self-consistent, similar coupled oscillation behavior of the charged particles can exist in an unbounded and self-consistently expanding plasma [30].

\section{Data Availability}

The data used to support the findings of this study are available from the corresponding author upon reasonable request.

\section{Conflicts of Interest}

The authors declare that they have no conflicts of interest.

\section{Acknowledgments}

This work was supported by the National Natural Science Foundation of China (Grant no. 11875091), the National K\&D Program of China (Grant no. 2016YFA0401100), and the Science and Technology on Plasma Physics Laboratory at CAEP.

\section{References}

[1] T. Nakazato, M. Oyamada, N. Niimura et al., "Observation of coherent synchrotron radiation," Physical Review Letters, vol. 63, p. 124, 1989.

[2] U. Happek, A. J. Sievers, and E. B. Blum, "Observation of coherent transition radiation," Physical Review Letters, vol. 67, no. 21, p. 2962, 1991.

[3] W. P. Leemans, C. G. R. Geddes, J. Faure et al., "Observation of terahertz emission from a laser-plasma accelerated electron bunch crossing a plasma-vacuum boundary," Physical Review Letters, vol. 91, Article ID 074802, 2003.

[4] R. L. Savage, C. Joshi Jr., and W. B. Mori, "Frequency upconversion of electromagnetic radiation upon transmission into an ionization front," Physical Review Letters, vol. 68, no. 7, p. 946, 1992.

[5] H. Hamster, A. Sullivan, S. Gordon, W. White, and R. W. Falcone, "Subpicosecond, electromagnetic pulses from intense laser-plasma interaction," Physical Review Letters, vol. 71 , no. 17 , p. $2725,1993$.

[6] H. Hamster, A. Sullivan, S. Gordon, and R. W. Falcone, "Shortpulse terahertz radiation from high-intensity-laser-produced plasmas," Physical Review E, vol. 49, no. 1, p. 671, 1994.

[7] G. L. Carr, M. C. Martin, W. R. Mckinney, K. Jordan, G. R. Neil, and G. P. Williams, "High-power terahertz radiation from relativistic electrons," Nature, vol. 420, no. 6912, p. 153, 2002. 
[8] P. Sprangle, J. R. Peñano, B. Hafizid, and C. A. Kapetanakos, "Ultrashort laser pulses and electromagnetic pulse generation in air and on dielectric surfaces," Physical Review E, vol. 69, Article ID 066415, 2004.

[9] T. P. Yu, Y. Y. Ma, W. W. Chang, Y. Yin, C. L. Tian, and F. Q. Shao, "Numerical simulation on effect of laser parameters on terahertz radiation," High Power Laser and Particle Beams, vol. 20, p. 943, 2008, in Chinese.

[10] Y. T. Li, C. Li, M. L. Zhou et al., "Strong terahertz radiation from relativistic laser interaction with solid density plasmas," Applied Physics Letters, vol. 100, Article ID 254101, 2012.

[11] P. Varshney, V. Sajal, P. Chauhan, R. Kumar, and N. K. Sharma, "Effects of transverse static electric field on terahertz radiation generation by beating of two transversely modulated Gaussian laser beams in a plasma," Laser and Particle Beams, vol. 32, p. 375, 2014.

[12] G. Q. Liao, Y. T. Li, Y. H. Zhang et al., "Demonstration of coherent terahertz transition radiation from relativistic lasersolid interactions," Physical Review Letters, vol. 116, Article ID 205003, 2016.

[13] G.-Q. Liao and Y.-T. Li, "Review of intense terahertz radiation from relativistic laser-produced plasmas," IEEE Transactions on Plasma Science, vol. 47, no. 6, p. 3002, 2019.

[14] K. Hu and L. Yi, "Relativistic terahertz radiation generated by direct-laser-accelerated electrons from laser-foil interactions," Physical Review A, vol. 102, Article ID 023530, 2020.

[15] L. Cao, W. Yu, H. Xu et al., "Terahertz radiation from oscillating electrons in laser-induced wake fields," Physical Review E, vol. 70, Article ID 046408, 2004.

[16] Z. M. Sheng, K. Mima, J. Zhang, and H. Sanuki, "Emission of electromagnetic pulses from laser wakefields through linear mode conversion," Physical Review Letters, vol. 94, Article ID 095003, 2005.

[17] W. M. Wang, P. Gibbon, Z. M. Sheng, and Y. T. Li, "Emission of electromagnetic pulses from laser wakefields through linear mode conversion," Physical Review Letters, vol. 114, p. 253901, 2015.

[18] A. Gopal, A. H. Woldegeorgis, S. Herzer et al., "Smith-Purcell radiation in the terahertz regime using charged particle beams from laser-matter interactions," Laser and Particle Beams, vol. 34, no. 1, p. 187, 2016.

[19] K. K. Magesh Kumar, M. Kumar, T. Yuan, Z. M. Sheng, and M. Chen, "Terahertz radiation from plasma filament generated by two-color laser gas-plasma interaction," Laser and Particle Beams, vol. 33, no. 3, p. 473, 2015.

[20] N. Ahmad, S. T. Mahmoud, G. Purohit, and F. Khan, "Two color laser self focusing and terahertz generation in multi-ion species plasma," Optik, vol. 158, p. 1533, 2018.

[21] G.-Y. Hu, B. Shen, A.-L. Lei, R.-X. Li, and Z.-Z. Xu, "Transition-Cherenkov radiation of terahertz generated by superluminous ionization front in femtosecond laser filament," Laser and Particle Beams, vol. 28, no. 3, p. 399, 2010.

[22] Y. Tian, J. Liu, Y. Bai et al., "Femtosecond-laser-driven wireguided helical undulator for intense terahertz radiation," Nature Photonics, vol. 11, no. 4, p. 242, 2017.

[23] S. Winner, E. Schomburg, S. Brand et al., "Frequency doubling and tripling of terahertz radiation in a gaas/alas superlattice due to frequency modulation of bloch oscillations," Applied Physics Letters, vol. 77, p. 1259, 2000.

[24] F. Bakhtiari, M. Esmaeilzadeh, and B. Ghafary, "Terahertz radiation with high power and high efficiency in a magnetized plasma," Physics of Plasmas, vol. 24, Article ID 073112, 2017.

[25] P. Rawat, V. Rawat, B. Gaur, and G. Purohit, "Generation of terahertz radiation by intense hollow Gaussian laser beam in magnetised plasma under relativistic-ponderomotive regime," Physics of Plasmas, vol. 24, Article ID 073113, 2017.

[26] R. K. Singh, M. Singh, S. K. Rajouria, and R. P. Sharma, "Strong terahertz emission by optical rectification of shaped laser pulse in transversely magnetized plasma," Physics of Plasmas, vol. 24, Article ID 073112, 2017.

[27] G. Purohit, V. Rawat, and P. Rawat, "Generation of terahertz radiation from beating of two intense cosh-Gaussian laser beams in magnetized plasma," Laser and Particle Beams, vol. 37, no. 4, p. 415, 2019.

[28] H. Xu, W. W. Chang, H. B. Zhuo, L. H. Cao, and Z. W. Yue, "Parallel programming of 2(1/2)-dimensional pic under distributed-memory parallel environments," Chinese Journal of Physics, vol. 19, p. 305, 2002, in Chinese.

[29] J. D. Jackson, Classical Electrodynamics, Chap. 14, Wiley, New York, NY, USA, 3rd edition, 1999.

[30] A. R. Karimov, L. Stenflo, and M. Yu, "Coupled azimuthal and radial flows and oscillations in a rotating plasma," Physics of Plasmas, vol. 16, Article ID 062313, 2009. 